\title{
Untersuchung einer neuen Herzklappe für Herzunterstützungssysteme
}

\author{
Lambertz M., Affeld K.*, Ziemann A., \\ * Labor für Biofluidmechanik, Universitätklinikum Charité, Humboldt-Universităt Berlin \\ Hermann-Föttinger Institut, Technische Universität Berlin
}

\section{EINLEITUNG}

Ein bewährtes Verfahren zur Behandlung erkrankter natïrlicher Herzklappen ist die Implantation von Herzklappenprothesen. Neben diesem Einsatz entwickelt sich zur Zeit ein weiteres Anwendungsgebiet: der Einsatz von Herzklappenprothesen in Herzunterstïtzungssystemen. Eine häufig dabei auftretende Komplikation stellt die Thrombenbildung dar [1]. Diese tritt insbesondere in Bereich der verwendeten Herzklappenprothesen auf [2]. Bei einer Implantation einer Herzklappenprothese ist ihre unmittelbare Umgebung durch die Anatomie vorgegeben. Bei einem Einsatz in einem Herzunterstützungssystem dagegen kann diese Umgebung frei gestaltet werden. Mit diesem Ansatz ergeben sich für die strömungstechnische Gestaltung der Herzklappen für Blutpumpen ganz neue Möglichkeiten. Die unmittelbare Umgebung der Klappe ist der Strömungskanal. Mit dem neuen, hier vorgestellten S-förmigen Strömungskanal der Klappe scheint es möglich zu sein, die Gefahr der Thrombenbildung zu reduzieren. Insbesondere die Vermeidung von Ablösungen führt zu einer Verringerung des Risikos der Thrombenbildung und ermöglicht damit eine verminderte Dosierung von Antikoagulantien

\section{Material UND Methode}

Die entwickelte S-Klappe besteht aus dem Strömungskanal, den Bügeln und dem Schließkörper. Durch den Sförmigen Strömungskanal, daher der Name S-Klappe, kann im Vergleich zu der Standard BS-Klappe auf einen Ring verzichtet werden.

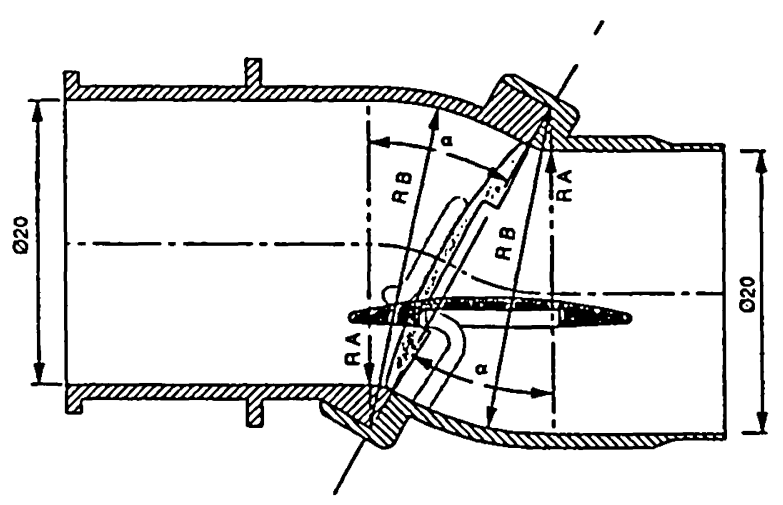

Abbildung1:Schema der Stromungskontur der S-Klappe.
Bei mechanischen Herzklappenprothesen sind die Ablösungen hinter dem Ring eine Ursache für die Thrombenbildung. Der SchlieBkörper steht im geschlossenem Zustand in einem Winkel von $60^{\circ}$ zur Anströmrichtung. Im geschlossenem Zustand liegt der SchlieBkörper in Anströmrichtung. Diese Positionen werden durch die Bügel vorgegeben. Die Form der Bügel entspricht den Bügeln der BS-Klappe. Ebenso ist dor Schließkörper der BS-Klappe entnommen. Der S-förmige Strömungskanal wird durch die Kombination verschiedener Radien erzeugt. Als Material für den Strömungskanal ist Titan vorgesehen. Bei der Untersuchung von Herzklappen werden die Ähnlichkeitsgesetze der Strömungsmechanik angewendet. Der sehr kurze Vorgang der Herzklappenströmung kann so zeitlich gestreckt beobachtet werden. Die Untersuchung erfolgt an 10:1 Modellen in einem gleichfalls vergrößerten Strömungskanal. Aus der Ähnlichkeitsbetrachtung ergibt sich mit einer angenommenen Blutviskosität von 2,7*10-3 Pas und einer Zykluszeit von $857 \mathrm{~ms}$ (Herzfrequenz=70min-1) eine Modellzykluszeit von tModell $=217,7 \mathrm{~s}$. Das Blut wird als Newtonesches Fluid betrachtet, weil die Scherraten ausreichend groß sind.

Für die Modelluntersuchung wird Wasser als Modellfluid gewählt. Der Viskositätsunterschied von Wasser und Blut wird in der Ähnlichkeitsbetrachtung berücksichtigt. Die Strömung durch die S-Klappe wurde mit folgenden Methoden der Strömungssichtbarmachung untersucht:

- Farbmethode mit fluoreszierendem Farbstoff

- Digital Particle Image Velocimetry (DPIV).

Bei der Strömungssichtbarmachung mit fluoreszierendem Farbstoff wird der Flüssigkeit im Raum hinter der Klappe mit einem Farbstoff eingefärbt. Der fluoreszierende Farbstoff wird während des Zyklus von dem ungefärbten Modellfluid verdrängt. Auf diese Weise lassen sich Gebiete mit hohen Verweilzeiten identifizieren. Diese Gebiete sind mitverantwortlich für die Thrombenbildung. Der fluoreszierende Farbstoff ist nur sichtbar, wenn er beleuchtet wird, und unsichtbar, wenn er im Dunklen liegt. Die Mitte der Strömung kann also durch entsprechende Beleuchtung mit einem Lichtschnitt beobachtet werden, obwohl der gesamte Raum mit Farbstoff gefüllt ist. Die zu untersuchenden Bereiche werden mit Hilfe von Diaprojektoren und entsprechenden Spaltblenden, die eine Lichtschnittdicke von circa $10 \mathrm{~mm}$ erzeugen, beleuchtet. Die Strömungssichtbar- 
machung erfolgt bei der Partikelmethode durch das Hinzufügen von kleinen Partikeln in die Strömung. Die Partikel folgen nahezu trägheitsfrei der Strömung. Damit entspricht die Bewegung der Partikel der Bewegung der zu untersuchenden Strömung. Auch bei dieser Methode wird der zu untersuchende Bereich mit einem Lichtschnitt ausgewählt. Die in dieser Ebene beleuchteten Partikel reflektieren das Licht und erscheinen als helle Punkte. Die Bewegung der Partikel wird mit einer Aufnahmeeinrichtung, hier einer CCD-Kamera, registriert. Die Bestimmung der lokalen Geschwindigkeit mit der Partikelmethode erfolgt durch die Erfassung der Partikelbewegung in einem Zeitintervall. Betrachtet man zwei Partikelbilder mit einem bekannten zeidlichen Abstand, befinden sich die Partikel bei einer vortandenen Strömung in den zwei Bildern an unterschiedlichen Orten. Durch Verbinden der Orte erhält man einen Bahnvektor. Mit der Zeitdifferenz zwischen den Bildern lassen sich Richtung und Größe der Geschwindigkeit bestimmen. Mit dem beschriebenen Meßprinzip sind nur Geschwindigkeitskomponenten parallel zum Lichtschnitt ermittelbar. Es handelt sich also hierbei um eine 2-dimensionale Geschwindigkeitsmessung. Dieses Verfahren wird als Digital Particle Image Velocimetry DPIV - bezeichnet. Bei der DPIV werden die Bilder direkt mit einer elektronischen Aufnahmeeinrichtung aufgenommen und digitalisiert. Die Speicherung der Bilder erfolgt im Speicher des angeschlossenen Rechners. Zur Bestimmung der Partikelverschiebung werien die aufgenommenen Bilder mit der Analysesoftware "VisiFlow" bearbeitet. Die Bildanalyse erfolgt mit der Kreuzkorrelation. Mit ihrer Hilfe kann die Verschiebung eines Musters, das in zwei aufeinanderfolgenden Bildern aufurtt, bestimmt werden. Mit dem bekannten zeitlichen Abstand der Bilder läßt sich somit cie Partikelgeschwindigkeit bestimmen.

\section{ERGEBNISSE}

Mit der Farbstoffmethode mit fluoreszierenden Farben konnten drei Ablösegebiete im Bereich der Klappenströmung beobachtet werden. Abbildung 2 zeigt cie Strömung durch die S-Klappe nach $t$ Modell $=22$ s. Diese hell erscheinenden Ablösegebiete lassen sich jedoch, wie Versuche mit einer geringfügig modifizierten Strömungskanal gezeigt baben, fast vollständig beseitigen. Die DPIV ermöglicht die vektorielle Beschreibung der Strömung. Beispielsweise lassen sich Öfnungsphase oder die ausgeprägte Strömung darstellen. In Abbildung 3 ist beispielhaft die ausgeprägte Strömung nach $\mathrm{tMod}=22 \mathrm{~s}$ zu sehen. Mit der in dieser Darstellung für die Bildanalyse gewăhlten Auflösung sind die Ablösegebiete nicht zu erkennen. Bei der Bildanalyse mit einer bobheren Auflosung lassen sich auch die Ablösegebiete darstellen. $\mathrm{Da}$ es hierbei jedoch im gesamten Stromungsbild zu einer Häufung von Fehlvektoren kommı, wird fur die Darstellung der gesamten Stromung eine geringe Aunosung gewahlt. Die Genauigkeit der Messung wird dadurch nicht beeinflußt.

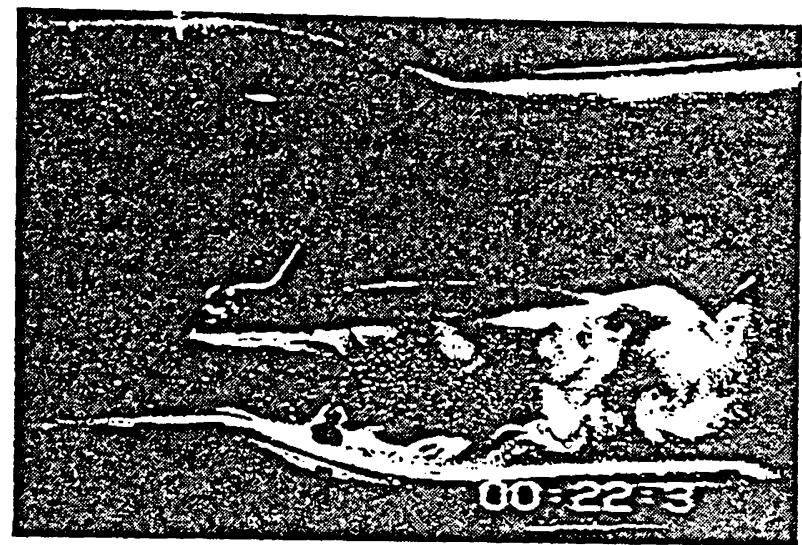

Abbildung 2: Farbstoffaufnahme der Stromung durch die S-Klappe nach 22s mit Lichtschnitt

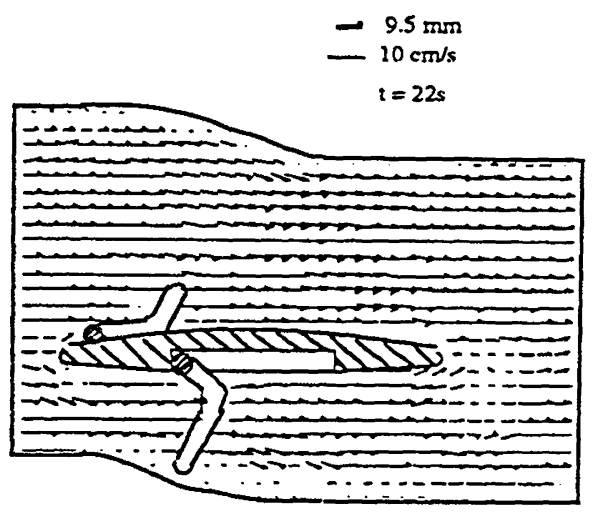

Abbildung 3: Vektorbild der Stromung nach 22 s

\section{ZUSAMMENFASSUNG}

An der neuentwickelten S-Klappe wurden ctrei Ablösegebiete beobachtet. Mit verschiedenen Modifizienungen konnten diese Ablösegebiete jedoch verringert bzw. vollständig beseitigt werden. Damit steht mit der modifizierten S-Klappe für den Einsatz in Herzunterstützungssystem eine Herzklappe zur Verfügung, die in ihrer Strömung keine Strömungsablösungen mehr aufweist. Die Untersuchung der Strömung läßt vermuten, daB die Thrombenbildung bei der modifizierten S-Klappe geringer als bei konventionellen Kippscheibenprothesen ist. Dies ist in Tierversuchen noch $z u$ verifizieren.

\section{LITERATUR:}

[1] Babatasi, G.; Massetti, M.; Bhoyron, A.; Khayat. A.: Clinical Experience with the Medos" Assist Device. Cardiovascular Eng., 1997, V.2., No. 1

[2] Wagner, W.R., Johnson, P.C. Kormos, R.L.; Griffith, B.P.: Evaluation of Bioprosthetic ValveAssociated Thrombus in Ventricular Assist Device Patients. Circulation, 1993, 88. S. 2023-2029 .

[3] Affeld, K., Gadischke, J.: Shear Rate and Thrombin Transpor, Procecdings of third World Congress for Biofluidmechanics. Munich 1994, VDI Verlag. Reihe 17, Biotechnik Nr. 107, r.35-40. 